\title{
Polymorphismes génétiques des apolipoprotéines
}

Le rôle des facteurs génétiques dans les hyperlipidémies et dans l'athérosclérose est à présent bien établi. Il a été très étudié en comparant la fréquence de marqueurs polymorphes liés aux apolipoprotéines ou à la fréquence des complications de l'athérosclérose. Le polymorphisme peut être détecté grâce à des différences de mobilité électrophorétique ou d'antigénicité des apolipoprotéines; il fait maintenant appel de plus en plus souvent à la démonstration de sites polymorphes dans ou à proximité des gènes d'apolipoprotéines. L'athérosclérose est manifestement un désordre impliquant plusieurs gènes. Au plan des populations, l'effet cumulé de plusieurs facteurs génétiques de prédisposition, individuellement peu délétères mais fréquents semble plus important que l'influence de mutations à forte pénétrance phénotypique, mais rares, telle celle du gène du récepteur des LDL dans l'hypercholestérolémie familiale.

\section{François Cambien}

\section{ADRESSE}

F. Cambien : docteur en médecine, directeurde recherche à l'Inserm. Inserm U.258 et SC7, hôpital Broussais, 96, rue Didot, 75674 Paris Cedex 14, France.

\section{TIRÉS A PART}

F. Cambien. l est à présent bien établi que le développement de l'athérosclérose et le niveau de ses facteurs de risque sont influencés par des mécanismes génétiques. Cette conviction se fonde sur les résultats d'une série de travaux épidémiologiques et cliniques [1] et est fortement renforcée par l'observation de maladies génétiques, telle l'hypercholestérolémie familiale, au cours desquelles le développement de l'athérosclérose est accéléré et le risque de maladie coronarienne considérablement accru. Cependant, ces maladies relativement rares et bien caractérisées ne sont observées que chez une petite fraction des sujets victimes d'un infarctus et il y a de fortes raisons de penser que des anomalies génétiques, mineures mais fréquentes, seules ou en association, ont un impact plus important sur l'incidence globale de cette pathologie [2].

\section{Apolipoprotéines et athérosclérose}

Les apolipoprotéines interviennent dans le déterminisme de la maladie coronarienne et leur taux circulant est en partie déterminé génétiquement. Les résultats de nombreuses études suggèrent que les apolipoprotéines plasmatiques sont des facteurs de risque majeurs de la maladie coronarienne [3, 4]. En outre, la ressemblance familiale du taux des lipoprotéines et des apolipoprotéines a été démontrée à plusieurs reprises et les résultats de différents travaux amènent à penser que le caractère familial de la pathologie coronarienne est largement déterminé par les facteurs 


\section{RÉFÉRENCES}

1. Goldbourt U, Neufeld HN. Genetic aspects of arteriosclerosis. Arteriosclerosis $1986 ; 6$ : $357-77$.

2. Davignon J, Gregg RE, Sing CF. Apolipoprotein E polymorphism and atherosclerosis. Arteriosclerosis 1988; 8 : 1-21.

3. Franzen J, Fex G. Low serum apolipoprotein A-I in acute myocardial infarction survivors with normal HDL cholesterol. Atherosclerosis $1986 ; 59: 37-42$

4. Sedlis SP, Schechtman KB, Ludbrook PA, Sobel BE, Schonfeld G. Plasma apoproteins and the severity of coronary artery disease. Circulation 1986; 73 : 978-86.

5. Freedman DS, Srinivasan SR, Shear CL, Franklin FA, Webber LS, Berenson GS. The relation of apolipoproteins A-I and B in children to parental myocardial infarction. $N$ Engl J Med 1986 ; 315 : 721-6.

6. Cambien F, Warnet JM, Jacqueson A Ducimetière P, Richard JL, Claude JR. Relation of parental history of early myocardial infarction to the level of apoprotein B in men. Circulation 1987 ; 76 : 266-71.

7. Nei M. Molecular evolutionary genetics. New York : Colombia University Press, 1987 254-86.

8. Brink PA, Steyn LT, Coetzee GA, Van der Westhuyzen DR. Familial hypercholesterolemia in South African afrikaners. PvuII and StuI DNA polymorphism in the LDL-receptor gene consistent with a predominating founder gene effect. Hum Genet. 1987; 77 : $32-5$.

9. Hobbs HH, Brown MS, Russel DW, Davignon J, Goldstein JL. Deletion in the gene for the low density lipoprotein receptor in a majority of French Canadians with familial hypercholesterolemia. N Engl J Med 1987; 317 : 734-7.

10. Ma Y, Schumaker VN, Butler R, Sparkes RS. Two DNA restriction fragment length polymorphism associated with $\mathrm{Ag}(\mathrm{t} / \mathrm{z})$ and $\mathrm{Ag}(\mathrm{g} / \mathrm{c})$ antigenic sites of human apolipoprotein B. Arteriosclerosis $1987 ; 7$ : 301-5.

11. Duriez P, Vu Dac N, Koffigan M, et al. Detection of human apolipoprotein B polymorphic species with one monoclonal antibody (BIP 45) against low density lipoprotein. Atherosclerosis $1987 ; 66: 153-61$.

12. Wang X, Schlapfer P, Ma Y, Butler R, Elovson J, Schumaker VN. Apolipoprotein $\mathrm{B}$ : the $\mathrm{Ag}(\mathrm{al} / \mathrm{d})$ immunogenetic polymorphism coincides with a $\mathrm{T}$ to $\mathrm{C}$ substitution at nucleotide 1981, creating an AluI restriction site. Arteriosclerosis 1988; 8 : 42935.

13. Thompson EA, Deeb S, Walker D, Motulsky AG. The detection of linkage disequilibrium between closely linked markers : RFLPs at the AI-CIII apolipoprotein genes. génétiques qui affectent le métabolisme des lipides [5, 6]. Ces constatations permettent de comprendre l'intérêt porté ces dernières années aux variants génétiques et aux polymorphismes des protéines impliquées dans le métabolisme des lipides.

Un polymorphisme est un caractère monogénique qui se transmet suivant le mode mendélien et qui est présent dans la population sous la forme d'au moins deux phénotypes dont la fréquence n'est pas rare. Conventionnellement, on parle de polymorphisme pour un phénotype dont la fréquence est supérieure à $1 \%$ et de variant dans le cas contraire. Les polymorphismes sont nombreux. Différents travaux ont permis de montrer que le pourcentage de nucléotides qui diffèrent au niveau d'une paire de séquences d'ADN homologues est chez l'homme de 0,2 à $0,3 \%$ [7].

Cette diversité, qui explique en grande partie l'individualité biochimique des individus, est probablement essentiellement neutre vis-à-vis de l'évolution, mais elle peut, dans certaines circonstances, avoir des conséquences défavorables et être un élément déterminant du développement des maladies chroniques. Ces maladies (athérosclérose, hypertension, diabète, cancer, maladies neurologiques et du vieillissement...) sont caractérisées entre autres par leur grande fréquence et leur survenue à un âge relativement tardif qui fait qu'elles n'interfèrent pas ou très peu avec les capacités reproductives des individus qui en sont victimes. Les gènes qui les favorisent peuvent donc être très fréquents et se retrouver simultanément chez un nombre non négligeable de sujets; de plus, l'effet de ces facteurs génétiques est modulé par des facteurs environnementaux, en particulier nutritionnels, ce qui explique que des populations vivant dans certaines conditions d'environnement puissent être indemnes de ces maladies, alors qu'elles peuvent en devenir victimes dès que le milieu devient défavorable. Ceci est par exemple le cas pour les anomalies des lipoprotéines, qui sont très dépendantes des caractéristiques de l'alimentation.

Il est donc possible qu'un certain nombre de polymorphismes généti- ques affectant les protéines impliquées dans le métabolisme des lipides jouent un rôle important dans le déterminisme de l'athérosclérose. Les résultats de plusieurs études réalisées ces dernières années tendent à confirmer cette hypothèse.

Les variants du récepteur des lipoprotéines de basse densité (LDL) responsables de l'hypercholestérolémie familiale (HCF) et le polymorphisme de l'apolipoprotéine $E$ (ApoE) : illustrations des concepts de risque individuel et de risque au niveau de la population.

- L'anomalie du récepteur des $L D L$ et l'hypercholestérolémie familiale. L'hypercholestérolémie familiale est une redoutable maladie monogénique qui est la conséquence d'une anomalie du récepteur de l'ApoB/E. Un grand nombre de variants du récepteur des LDL ont été décrits qui résultent d'au moins 16 délétions différentes au niveau du zène. Si bien que la majorité des sujets dits homozygotes sont en fait des hétérozygotes composites. Dans certaines populations, cette affection est fréquente; c'est le cas dans la population Afrikaner [8] où la forme hétérozygote est présente chez un sujet sur 100. Le pool génétique des Afrikaners est relativement petit. Au cours d'une période d'environ 300 ans, le nombre d'individus composant cette population a été multiplié par plusieurs milliers, alors que, dans le même temps, la population européenne était multipliée par six. De plus existait une consanguinité importante au sein des quelques communautés créées par ces migrants. Il a été montré qu'il suffisait d'un ou de deux gènes défectifs dans la population d'origine pour expliquer la très grande fréquence de l'anomalie observée actuellement. L'identité de la lésion génétique à l'origine de l'HCF chez la majorité des Afrikaners n'a pas été rigoureusement établie ; néanmoins, l'anomalie est en règle générale transmise en même temps qu'un polymorphisme de restriction défini par les enzymes StuI et PvuII du gène du récepteur des LDL, ce qui suggère fortement un effet fondateur (en effet si l'anomalie ne provenait pas du même fondateur, les allèles associés à la maladie pour- 
raient varier d'une famille à l'autre). Une situation à peu près similaire existe dans un sous-groupe de la population libanaise et chez les Canadiens français. Chez ces derniers aussi on observe une fréquence importante de l'HCF (moins importante toutefois que chez les Afrikaners) et il a été possible d'établir que $60 \%$ des patients présentent une délétion de plus de $10 \mathrm{~kb}$ au niveau de l'exon 1 du gène du récepteur des LDL [9].

En dehors de ces populations particulières, la fréquence des formes hétérozygotes est d'environ 1/500 et celle des formes homozygotes ou hétérozygotes composites est de un sur un million.

- Le polymorphisme électrophorétique de l'apolipoprotéine $E$ $(A p o E)$ [2]. L'ApoE a une grande affinité pour le récepteur $\mathrm{B} / \mathrm{E}$ hépatique et joue un rôle déterminant dans le métabolisme des lipoprotéines de densité très basse (VLDL) et intermédiaire (IDL). Plusieurs variants de l'ApoE ont été décrits, mais ce qui est particulièrement notable, c'est l'existence de deux sites polymorphes dont les allèles ont des fréquences importantes dans la population. La forme sauvage de la protéine, appelée $\varepsilon 3$, porte une cystéine en position $112 \mathrm{et}$ une arginine en position 158 dans la région de reconnaissance du récepteur de l'ApoB/E. La forme $\varepsilon 2$ porte une cystéine en position 158 et la forme $\varepsilon 4$ porte une arginine en position 112. La forme $\varepsilon 2$ a une affinité pour le récepteur des apolipoprotéines $\mathrm{B} / \mathrm{E}$ réduite, ce qui induit, en particulier chez les sujets homozygotes $\varepsilon 2 \varepsilon 2$, des anomalies du métabolisme des VLDL et des IDL avec, principalement, une augmentation de la quantité circulante de ces lipoprotéines et une diminution des lipoprotéines de basse densité (LDL) due probablement à une augmentation du nombre des récepteurs hépatiques des LDL. Les individus $\varepsilon 4 \varepsilon 4$, au contraire, ont des VLDL abaissées et des LDL augmentées par rapport aux autres sujets.

Le Tableau I rapporte les fréquences relatives des trois allèles communs dans un certain nombre d'études épidémiologiques. Malgré l'assez grande homogénéité des fréquences entre études, des différences méritent $\mathrm{m} / \mathrm{s} n^{\circ} 6$ vol. 5 , juin 89

\begin{tabular}{|l|r|rrr|}
\hline \multicolumn{5}{|c|}{$\begin{array}{c}\text { Tableau I } \\
\text { POLYMORPHISME DE LAPoE DANS } \\
\text { DIFFÉRENTES POPULATIONS }\end{array}$} \\
\hline \hline \multicolumn{3}{|c|}{ Population } & Effectifs & Fréquences relatives \\
\cline { 3 - 6 } & & $\varepsilon 2$ & $\varepsilon 3$ & $\varepsilon 4$ \\
\hline Japonais & & & & \\
Asahikawa & 576 & 0,037 & 0,846 & 0,117 \\
Hiroshima Nagasaki & 110 & 0,023 & 0,891 & 0,086 \\
Tokyo & 197 & 0,038 & 0,843 & 0,112 \\
\hline Caucasiens & & & & \\
Christchurch (Nouvelle Zélande) & 426 & 0,120 & 0,720 & 0,160 \\
Framingham (USA) & 1209 & 0,072 & 0,786 & 0,140 \\
Grampian (Écosse) & 400 & 0,083 & 0,770 & 0,147 \\
Helsinki (Finlande) & 615 & 0,041 & 0,733 & 0,227 \\
Marburg (RFA) & 1031 & 0,077 & 0,773 & 0,150 \\
Munich (RFA) & 1557 & 0,082 & 0,782 & 0,136 \\
Nancy (France) & 223 & 0,130 & 0,742 & 0,128 \\
\hline \hline
\end{tabular}

Voir Davignon et al. [2] pour connaître les références de ces différentes études.

d'être notées: en particulier, la fréquence relativement basse des allèles $\varepsilon 2$ et $\varepsilon 4$ chez les Japonais, population à bas risque d'athérosclérose, et la fréquence importante de l'allèle $\varepsilon 4$ chez les Finlandais, population à haut risque d'athérosclérose. Les effets respectifs associés à la présence d'un de ces trois allèles sur le taux de cholestérol sont extrêmement homogènes d'une étude à l'autre; l'allèle \&3 apparaît neutre, alors que $\varepsilon 2$ est associé à une baisse de 0,25 à $0,50 \mathrm{mmol} / 1 \mathrm{du}$ taux de cholestérol et que $\varepsilon 4$ est associé à une augmentation de 0,12 à $0,25 \mathrm{mmol} / 1$ de ce taux. Les effets alléliques paraissent grossièrement additifs comme le montre le Tableau II ( $p .382)$ où les moyennes des taux de cholestérol suivant les six phénotypes possibles sont donnés pour un certain nombre d'études épidémiologiques. Ainsi, il apparaît que les sujets porteurs du phénotype $\varepsilon 4 \varepsilon 4$ ont en moyenne un taux de cholestérol 0,75 à $1,0 \mathrm{mmol} / \mathrm{l}$ au-dessus de celui des sujets de phénotype $\varepsilon 2 \varepsilon 2$. Cette différence peut paraître modeste, surtout si on la compare à celle associée avec l'anomalie du récepteur des LDL qui est à l'origine de l'HCF, mais elle induit un doublement du risque d'infarctus du myocarde chez un nombre non négligeable de sujets. En effet 2,25\% de la population caucasienne porte le phénotype $\varepsilon 4 \varepsilon 4$; ce pourcentage s'élève même à $5 \%$ dans la population finlandaise, soit une fréquence 25 fois plus grande que l'HCF.

Le polymorphisme de l'ApoE est donc d'un grand intérêt épidémiologique. Il pourrait n'affecter que légèrement le risque individuel, mais sa grande fréquence dans la population pourrait avoir un profond impact sur la pathologie. On estime qu'il explique $7 \%$ de la variabilité de la cholestérolémie dans la population, alors que les variants du récepteur des LDL associés à l'HCF n'en expliquent que moins de $1 \%$. Cela illustre bien les implications différentes sur le risque individuel et sur le risque 


\section{RÉFÉRENCES}

14. Kessling AM, Nanjee MN, Miller NE, Humphries SE. Variations in the apolipoprotein AI-CIII-AIV gene region and in lecithin : cholesterol acyltransferase concentration are determinants of plasma cholesterol concentrations. Atherosclerosis 1988 ; 70 : 13-9.

15. Li WH, Tanimura M, Luo CC, Daha S, Chan L. The apolipoprotein multigene family : biosynthesis, structure-function relationships and evolution. J Lipid Res 1988; 29 : 245-72.

16. Klasen EC, Talmud PJ, Havekes. L. A common restriction fragment length polymorphism of the human apolipoprotein $\mathbf{E}$ gene and its relationship with type III hyperlipidaemia. Hum Genet 1988; 75 : 244-7.

17. Humphries SE. DNA polymorphisms of the apolipoprotein genes. Their use in the investigation of the genetic component of hyperlipidaemia and atherosclerosis. Atherosclerosis $1988 ; 72: 89-108$.

18. Rees A, Shoulders CC, Stocks J, Galton DJ, Baralle FE. DNA polymorphism adjacent to human apoprotein $\mathrm{Al}$ gene : relation to hypertriglyceridemia. Lancet 1983 ; 1 . 444-6.

19. Rees A, Stocks J, Sharpe CR, et al., Deoxyribonucleic acid polymorphism in the apolipoprotein Al-C3 gene cluster. J Clin Invest $1985 ; 76: 1090-5$

20. Ferns GAA, Stocks J, Ritchie C, Galton DJ. Genetic polymorphisms of apolipoprotein CIII and insulin in survivors of myocardial infarction. Lancet $1985 ; 2$ : 300-3.

21. Deeb S, Failor A, Brown BG, Brunzell JD, Albers JJ, Motulsky AG. Molecular genetics of apolipoproteins and coronary heart disease. In Cold Spring Harbor symposia on quantitative biology, vol. LI. Cold Spring Harbor: Cold Spring Harbor Laboratory, 1986 ; 403-9.

22. Rees A, Stocks J, Paul H, Ohuchi Y, Galton D. Haplotypes identified by DNA polymorphisms at the apolipoprotein $\mathrm{Al}$ and CIII loci and hypertriglyceridemia. A study in a Japanese population. Hum Genet 1986 ; 72 : 168-71.

23. Trembath RC, Hendra TJ, Thomas DJB, Yudkin JS, Galton DJ. Deoxyribonucleic acid polymorphism of the apoprotein AI-CIIIAIV gene cluster and coronary heart disease in non-insulin-dependent diabetes. $\mathrm{Br} \mathrm{Med} J$ $1987 ; 294: 1577-8$

24. Henderson HE, Landon SV, Michie J, Berger GMB. Association of a DNA polymorphism in the apolipoprotein CIII gene with diverse hyperlipidaemic phenotypes. Hum Genet 1987 ; 75 : 62-5.

25. Aalto-Setälä K, Kontula K, Sane T, Nieminen M, Nikkilä E. DNA polymorphisms of apolipoprotein AI/CIII and insulin in familial hypertriglyceridemia and coronary heart

\begin{tabular}{|c|c|c|c|c|c|c|}
\hline \multicolumn{7}{|c|}{$\begin{array}{c}\text { Tableau ॥ } \\
\text { CONCENTRATION PLASMATIQUE DU CHOLESTÉROL EN FONCTION } \\
\text { DES DIFFÉRENTS PHÉNOTYPES DE L'APOE }\end{array}$} \\
\hline \multirow[b]{2}{*}{ Population } & \multicolumn{6}{|c|}{ Taux de cholestérol moyen (mg/dl) } \\
\hline & $\varepsilon 2 / \varepsilon 2$ & $\varepsilon 2 / \varepsilon 3$ & $\varepsilon 2 / \varepsilon 4$ & $\varepsilon 3 / \varepsilon 3$ & $\varepsilon 3 / \varepsilon 4$ & $\varepsilon 4 / \varepsilon 4$ \\
\hline Asahikawa (Japon) & 142 & 164 & 214 & 174 & 178 & 180 \\
\hline Framingham (USA) & 184 & 195 & 214 & 210 & 217 & 214 \\
\hline Helsinki (Finlande) & 173 & 202 & 196 & 214 & 223 & 239 \\
\hline Marburg (RFA) & 140 & 166 & & 184 & 189 & 197 \\
\hline Munich (RFA) & 178 & 182 & 189 & 195 & 199 & 203 \\
\hline Nancy (France) & 199 & 207 & 237 & 235 & 241 & 240 \\
\hline
\end{tabular}

Voir Davignon et al. [2] pour connaître les références de ces différentes études.

collectif des variants génétiques rares et des polymorphismes fréquents et permet de comprendre la nécessité d'une approche épiłémiologique de la relation entre polymorphisme génétique et pathologie lipidique ou coronarienne.

- Interactions entre gènes: hyperlipidémie de type III (voir glossaire, p. 388) et phénotype ع2ع2. $90 \%$ des individus porteurs d'une hyperlipidémie de type III ont le phénotype $\varepsilon 2 \varepsilon 2$. La fréquence de cette maladie extrêmement athérogène est d'environ $1 / 10000$ dans la population, soit environ $1 / 100$ des sujets $\varepsilon 2 \varepsilon 2$. L'hyperlipidémie de type III est ainsi probablement la conséquence d'une interaction entre la diminution d'affinité des particules lipoprotéiques portant $\varepsilon 2$ pour le récepteur des Apo $\mathrm{B} / \mathrm{E}$ et une ou plusieurs autres anomalies d'origine génétique ou environnementale et qui, pour l'instant, ne sont pas connues. Les phénomènes d'interaction entre gènes (l'effet d'un gène modifie l'effet d'un autre gène) ou entre gène et environnement (l'effet d'un gène modifie l'effet d'un facteur d'environnement ou inversement) sont susceptibles d'induire des effets phénotypiques tout à fait subtils et parfois apparemment paradoxaux. Ainsi, dans le cas présent, une caractéristique génétique, le phénotype $\varepsilon 2 \varepsilon 2$, s'accompagne d'un effet globalement bénéfi- que, mais lorsqu'elle est associée à un autre facteur rare et pour l'instant inconnu, elle a un effet délétère.

- Polymorphisme immunochimique des LDL. Cinq sites polymorphes distincts situés dans la partie codante du gène de l'ApoB déterminent le polymorphisme immunochimique des LDL connu sous le nom de système Ag. Cinq paires d'allèles ont été identifiées : $\mathrm{Ag}(\mathrm{al} / \mathrm{d}), \mathrm{Ag}(\mathrm{x} / \mathrm{y}), \mathrm{Ag}(\mathrm{t} / \mathrm{z}), \mathrm{Ag}(\mathrm{g} / \mathrm{c})$ et $\mathrm{Ag}(\mathrm{h} / \mathrm{i})$. L'étude de ces polymorphismes en fonction des taux de lipoprotéines et d'ApoB et de la présence ou non de pathologie coronarienne n'a pas permis de dégager d'associations très fortes. Néanmoins, certains résultats montrent une association entre $\mathrm{Ag}(\mathrm{x} / \mathrm{y})$ et les taux de triglycérides et de cholestérol. Cette association est cohérente avec l'observation d'une association entre ce polymorphisme et le polymorphisme de restriction XbaI, qui luimême a été trouvé à plusieurs reprises associé à la concentration de lipides. Des résultats récents indiquent que les polymorphismes $\mathrm{Ag}$ $(\mathrm{x} / \mathrm{y})$ et $\mathrm{Ag}(\mathrm{c} / \mathrm{g})$ sont situés à proximité l'un de l'autre [10] et il est notable que l'épitope reconnu par un anticorps monoclonal, le Bip 45, est également situé dans cette région [11]. Le séquençage nucléotidique de la région a permis de montrer que la mutation en cause dans 
le polymorphisme XbaI affecte la troisième base d'un codon thréonine correspondant au résidu 2488 de la protéine mature. Du fait de son caractère silencieux, cette mutation n'est donc pas celle mise en évidence par le Bip 45 ou l'anticorps anti-Ag (x/y).

Le polymorphisme $\mathrm{Ag}(\mathrm{t} / \mathrm{z})$ est quant à lui la conséquence d'une substitution Glu $\rightarrow$ Lys au niveau du résidu 4154 de la protéine mature [10] et le polymorphisme $\mathrm{Ag}(\mathrm{al} / \mathrm{d})$ est dû à une substitution Val $\rightarrow$ Ala en position 59l de la protéine [12]. Cette mutation résulte d'une substitution $\mathrm{T} \rightarrow \mathrm{C}$ au niveau de la base $1981 \mathrm{du}$ gène de l'ApoB, qui crée un site de restriction AluI (AGTT $\rightarrow$ AGCT). La mutation est donc aisément identifiable au niveau génomique en utilisant une enzyme reconnaissant la séquence AGCT et une courte sonde synthétique.

Les anticorps monoclonaux peuvent être des outils très performants pour étudier le polymorphisme génétique des apolipoprotéines. A l'heure actuelle, c'est surtout l'ApoB qui a été étudiée par cette approche. Certains anticorps reconnaissent des épitopes se trouvant au niveau du site de fixation de l'ApoB sur son récepteur. Ils permettent donc de repérer des régions très précises qui peuvent être étudiées plus en détail par les techniques de biologie moléculaire.

\section{Polymorphismes de restriction des gènes des apolipoprotéines}

La méthode de Southern (voir $\mathrm{m} / \mathrm{s}$, lexique, suppl. au $n^{\circ} 7$, vol. 3, p. 2) a été très utilisée ces dernières années pour la recherche de polymorphismes génétiques. Le Tableau III résume les principaux polymorphismes mis en évidence par cette technique au niveau des gènes impliqués dans le métabolisme des lipo-

Tableau III

PRINCIPAUX POLYMORPHISMES DE RESTRICTION DANS LE DOMAINE DU MÉTABOLISME DES LIPIDES

\begin{tabular}{|c|c|c|c|c|c|}
\hline & АpoA1/C3/A4 & ApoC1/C2/E & ApoB & LDLR & \\
\hline Enzyme & \multicolumn{5}{|c|}{ Fréquence de l'allèle le plus rare (région identifiée par la sonde)* } \\
\hline $\begin{array}{l}\text { Apal } \\
\text { ApaLI } \\
\text { Aval } \\
\text { Avall } \\
\text { Banl } \\
\text { BanII } \\
\text { BamHI } \\
\text { BcII } \\
\text { BgII } \\
\\
\text { BgIII } \\
\text { Bsml } \\
\text { BstEII } \\
\text { BstNI } \\
\text { Dral } \\
\text { EcoRI } \\
\text { EcoRV } \\
\text { Hincll } \\
\text { HindIII } \\
\text { Hpal } \\
\text { Mspl } \\
\text { Ncol } \\
\text { PstI } \\
\text { Pvull } \\
\\
\text { Rsal } \\
\text { SstI } \\
\text { Stul } \\
\text { Taql } \\
\text { Xbal } \\
\text { Xmnl }\end{array}$ & $\begin{array}{l}0,33 \text { (A4-3') } \\
0,03 \text { (C3) } \\
0,19 \text { (A4) } \\
0,05 \text { (A1) } \\
\\
0,12 \text { (A1) } \\
0,3 \text { (A4) } \\
0,06 \text { (A1-3') } \\
\text { a) } 0,3 \text { (C3) } \\
\text { b) } 0,07 \text { (A4-5') } \\
\text { c) } 0,07 \text { (A4-3') } \\
0,13 \text { (C3) } \\
\text { a) } 0,28 \text { (A1) } \\
\text { b) } 0,02 \text { (A4) } \\
\text { a) } 0,16 \text { (a4) } \\
\text { b) } 0,25 \text { (A4-3') } \\
0,2 \text { (A1-5') }\end{array}$ & $\begin{array}{c}0,45(C 2) \\
0,3\left(C 2-3^{\prime}\right) \\
0,5\left(C 2-5^{\prime}\right) \\
0,02(C 1)\end{array}$ & $\begin{array}{c}0,2 \\
0,02 \\
0,12\left(B^{\prime}-5^{\prime}\right) \\
0,15 \\
0,08\left(B-5^{\prime}\right)\end{array}$ & $\begin{array}{l}0,23 \\
0,36 \\
0,4 \\
0,2 \\
\\
0,15 \\
0,06\end{array}$ & $\begin{array}{c}0,3 \text { (LPL) } \\
0,33 \text { (LPL) } \\
0,2(\text { ApoA2-3') } \\
0,41 \text { (LPL) }\end{array}$ \\
\hline
\end{tabular}

CETP : cholesteryl ester transfer protein; LPL: lipoprotein lipase. * Par exemple, A1-5' signifie en 5'du gène de l'ApoA1.

** Ces polymorphismes concernent Taql. 


\section{RÉFÉRENCES}

26. Hayden MR, Kirk H, Clark C. et al. DNA polymorphisms in and around the ApoAI-CIII genes and genetic hyperlipidemias. Am J Hum Genet 1987 ; 40 : 421-30.

27. Satoh J, Hattori N, Onuki M, et al. Apolipoprotein AI-CIII gene polymorphisms in Japanese myocardial infarction survivors. Jpn J Hum Genet 1987 ; 32 : 15-20.

28. Paulweber B, Friedl W, Krempler F, Humphries S, Sandhofer F. Genetic variation in the apolipoprotein AI-CIII-AIV gene cluster and coronary heart disease. Atherosclerosis $1988 ; 73: 125-33$

29. Ordovas JM, Schaeffer EJ, Salem D, et al. Apolipoprotein $\mathrm{Al}$ gene polymorphism associated with premature coronary artery disease and familial hypoalphalipoproteinemia. $N$ Engl J Med 1986; 314 : 671-7.

30. Kessling AM, Rajput-Williams J, Bainton $\mathrm{D}$, et al. DNA polymorphisms of the apolipoprotein AII and AI-CIII-AIV genes : A study in men selected for differences in highdensity-lipoprotein cholesterol concentration. Am J Hum Genet 1988; 42 : 458-67.

31. Scott J, Knott T J, Priestley LM, Robertson ME. High density lipoprotein composition is altered by a common DNA polymorphism adjacent to apolipoprotein AII gene in man. Lancet $1985 ; 1$ : 771-3.

32. Hegele RA, Huang L, Herbert PN, et al. Apolipoprotein B gene DNA polymorphisms associated with myocardial infarction. $N$ Engl J Med 1986 ; 315 : 1509-15.

33. Berg K. DNA polymorphism at the apolipoprotein B locus is associated with lipoprotein level. Clin Genet 1986 ; 30 : 515-20.

34. Law A, Wallis SC, Powell LM, et al. Common DNA polymorphism within coding sequence of apolipoprotein $B$ gene associated with altered lipid levels. Lancet 1986; 1 : 1301-3.

35. Talmud PJ, Barni N, Kessling AM, et al. Apolipoprotein B gene variants are involved in the determination of serum cholesterol levels : a study in normo and hyperlipidaemic individuals. Atherosclerosis 1987 ; 67 : 81-9.

36. Ferns GAA, Stocks J, Ritchie C, Galton DJ. Genetic polymorphisms of apolipoprotein CIII and insulin in survivors of myocardial infarction. Lancet. $1985: 2: 300-3$.

37. Aburatani $\mathrm{H}$, Matsumoto A, Murase $\mathrm{T}$, Itakura H. Apolipoprotein gene polymorphisms in Japanese. Arteriosclerosis $1987 ; 7$. 520 a.

38. Monsalve MV, Young R, Jobsis J, et al. DNA polymorphisms of the gene for apolipoprotein B in patients with peripheral arte rial disease. Atherosclerosis 1988 : 70 : 123-9.

39. Paul H, Galton D, Stocks J. DNA polymorphic patterns and haplotype arrangements of the ApoAl, ApoC3, ApoA4 gene cluster in different ethnic groups. Hum Genet $1987 ; 75: 264-8$ protéines. L'identification de ces polymorphismes permet de réaliser deux types d'études; d'une part, des études familiales qui visent à mettre en évidence des liaisons génétiques et, d'autre part, des études de population qui ont pour but de trouver des associations entre polymorphismes et maladie.

Les études familiales-études de liaison. Généralement, les polymorphismes de restriction sont étudiés dans des familles pour rechercher leur coségrégation avec un trait pathologique. En présence d'une telle coségrégation, qui reflète une liaison génétique, il peut être possible d'impliquer une région particulière du génome dans le déterminisme de la maladie ou, à l'inverse, d'exclure la responsabilité d'un gène particulier dans la maladie. Curieusement, cette approche - théoriquement très puissante et qui a été couronnée de succès dans d'autres domaines de pathologie et dans l'étude de la coségrégation de l'hypercholestérolémie familiale avec certains polymorphismes du récepteur des LDL - n'a pas encore eu d'impact très fort dans le domaine des apolipoprotéines. Cela est probablement dû au fait que la très grande majorité des dyslipoprotéinémies à composante génétique ne sont pas monogéniques. L'approche familiale est alors beaucoup plus complexe et nécessite des familles de très grande taille. On comprend alors que la quasi-totalité des études rapportées dans le domaine soient des études d'association.

Les études de population-études d'association. Une association entre un polymorphisme de restriction (utilisé comme marqueur) et un variant responsable d'une maladie génétique peut être détectée par des études de population, à condition qu'existe entre eux un déséquilibre de liaison. Ces études consistent en général à comparer les fréquences du polymorphisme dans un groupe de malades et un groupe de témoins ou à rechercher des associations entre le polymorphisme et des phénotypes intermédiaires (taux de certaines protéines, activités enzymatiques, nombres de récepteurs) dans des populations non malades.
Un déséquilibre de liaison est responsable d'une association au niveau de la population entre deux loci situés près l'un de l'autre. Il s'agit d'un phénomène fréquent. Conformément à la théorie classique, en l'absence de sélection, d'hétérogénéité de la population ou d'autres facteurs de confusion, le déséquilibre de liaison entre deux loci serait directement proportionnel à la distance physique les séparant [13], avec des paliers possibles.

De tels déséquilibres de liaison impliquant des polymorphismes de restriction ont été observés dans les régions des gènes des apolipoprotéines: les polymorphismes banI, TaqI et BglI au locus de l'ApoCII; XbaI et EcoRI au locus de l'ApoB; EcoRI, BamHI, MsPI, EcoRV également au locus de l'ApoB; MspI et SstI au locus de l'ApoAI-CIII ; TaqI et BglI au locus de l'ApoCII.

En théorie, plus grand est le degré de déséquilibre de liaison dans la région d'un caractère génétique supposé favorisant la maladie, plus grande est la possibilité de trouver un marqueur génétique de la maladie; de plus, l'étude d'haplotypes prenant en compte plusieurs polymorphismes de restriction est susceptible de mettre en évidence des associations beaucoup plus fortes que leur étude séparée. Par exemple, une étude a porté sur le taux de cholestérol HDL et d'ApoAI en fonction d'une série d'haplotypes définis par les enzymes de restriction XmnI, PstI et SstI du complexe ApoAI-ApoCIII [14]. Les associations mises en évidence se sont avérées beaucoup plus fortes que lorsque le polymorphisme PstI était étudié isolément. Une telle approche est très justifiée théoriquement dans la mesure où elle permet en quelque sorte d'obtenir des polymorphismes multialléliques, alors que l'une des limitations des polymorphismes de restriction est qu'ils sont en général bialléliques et donc peu informatifs.

Les gènes des apolipoprotéines AI, AII, AIV, CI, CII, CIII et E dérivent d'un même gène ancestral [15]. Les informations recueillies concernant la séquence et la structure des gènes codant pour les apolipoprotéines AI, AII, AIV, CI, CII, CIII et E suggèrent qu'ils dérivent d'un même gène ancestral. En effet, tous les gènes 


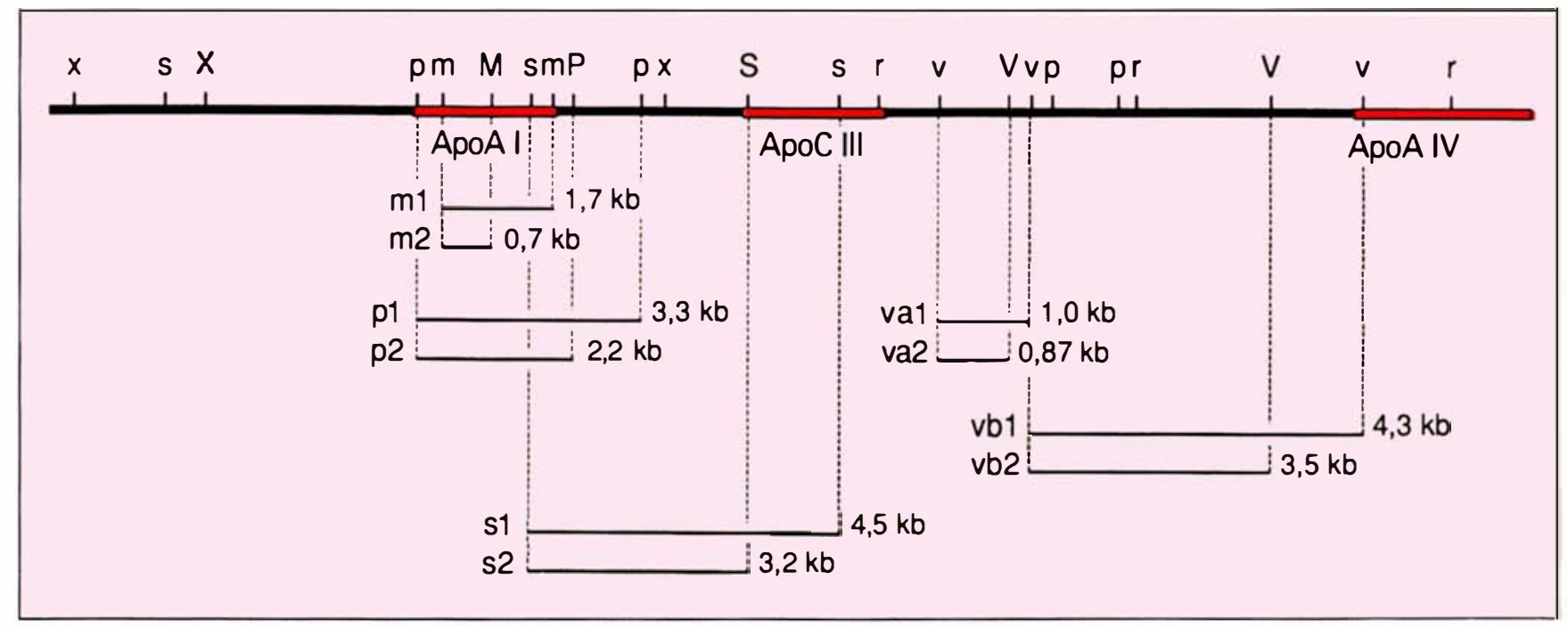

formant cette famille sauf celui de l'ApoAIV sont composés de quatre exons et de trois introns, et d'importantes analogies de séquence existent entre eux. De plus ces gènes, sauf un, sont groupés en deux complexes, l'un sur le chromosome 19 comprend les gènes des ApoE, CI et CII, l'autre sur le bras long du chromosome 11 comprend les gènes des ApoAI, CIII et AIV. Le gène de l'ApoAII est, quant à lui, isolé sur le chromosome $\mathrm{l}$.

Le complexe ApoE-CI-CII. Le rôle de l'ApoE a déjà été décrit précédemment. Le rôle de l'ApoCI est moins clair, cette apolipoprotéine est probablement impliquée dans l'activation de l'enzyme lécithine cholestérol acyltransférase (LCAT). Quant à l'ApoCII, c'est un activateur de la lipoprotéine lipase. Un certain nombre de patients porteurs d'une hyperlipidémie de type I ont un déficit en ApoCII, la maladie est récessive au niveau du phénotype lipoprotéique, bien que les hétérozygotes aient un taux circulant d'ApoCII de moitié inférieur à la normale. L'hyperlipidémie de type I peut également être observée chez les patients déficients en lipoprotéine lipase. Il est important de noter que les patients porteurs d'une hyperlipidémie de type I, malgré des taux de triglycérides considérables, ne souffrent en général pas d'athérosclérose.

Un polymorphisme $\mathrm{HpaI}$ proche du gène de l'ApoE a été décrit [16] dont l'allèle rare est présent chez $38 \%$ des sujets normolipidémiques. En revanche, cet allèle est retrouvé chez $\mathrm{m} / \mathrm{s} n^{\circ} 6$ vol. 5 , juin 89

Figure 1. Un exemple de déséquilibre de liaison entre polymorphismes de restriction. Sur cette figure sont indiqués les principaux sites de restriction trouvés au niveau du complexe génique ApoAl-CIII-AIV. Les sites non polymorphes sont symbolisés par une lettre minuscule et les sites polymorphes par une lettre majuscule. Les enzymes de restriction qui révèlent un polymorphisme sont : Xmnl $(x)$, Sstl (s), Pstl (p), Mspl (m), EcoRI (r), Pvull (v). Les fragments identifiables par la méthode de Southern sont indiqués au-dessous des sites correspondants. Les fréquences respectives des polymorphismes Mspl et Sstl chez les Caucasiens sont pour $M s p l: M 1=0,89$ et $M 2=0,11$, et pour $S s t l: S 1=0,91$ et $S 2=0,09$. En absence de déséquilibre de liaison, les fréquences des phénotypes M1S1, M2S1, M1S2, M2S2 devraient être respectivement : de 0,81, 0,10,0,08 et 0,01. Or les valeurs observées sur des effectifs importants sont: $0,879,0,004,0,007$ et 0,110 [13]. La différence entre les effectifs attendus et observés est très significative et montre une association (un déséquilibre de liaison) quasi complète entre M1 et S1, d'une part, et M2 et S2, d'autre part. On est donc en droit de penser que la région de $4 \mathrm{~kb}$ comprise entre ces deux polymorphismes est également en déséquilibre de liaison avec eux. Or le polymorphisme Pstl situé dans cette région n'est associé ni avec l'un ni avec l'autre. Les fréquences des deux allèles caractérisant ce polymorphisme sont : $P 1=0,93$ et $P 2=0,07$, et les fréquences des différents haplotypes impliquant les polymorphismes Pstl et Mspl d'une part et Pstl et Sstl d'autre part ne diffèrent pas des fréquences attendues sous I'hypothèse d'absence d'association; en particulier, $P 2 M 2=0,005$ et $P 2 S 2=$ 0,002 . Ce paradoxe résulte du fait que l'allèle rare Pstl est associé aux allèles fréquents Mspl et Sstl. Dans une telle situation, c'est-à-dire lorsqu'un allèle rare est associé à un allèle fréquent, la mise en évidence d'un déséquilibre de liaison nécessite des effectifs considérables.

$97 \%$ des patients ayant une dyslipidémie de type III. Il semble exister un déséquilibre de liaison quasi complet entre le polymorphisme $\mathrm{HpaI}$ qui est situé entre le gène de l'ApoE et celui de l'ApoCI et l'isoforme $\varepsilon 2$ de l'ApoE.

Le complexe ApoAI-CIII-AIV. L'ApoAI est le principal composant protéique des lipoprotéines de haute densité (HDL), elle est un cofacteur pour l'enzyme LCAT et pourrait se lier au récepteur des HDL. La fonction de l'ApoAIV, composant majeur des chylomicrons, est moins bien connue. Quant à l'ApoCIII, on la trouve sur les HDL et sur les VLDL; dans la mesure où elle inhibe l'activité de la lipoprotéine lipase, elle a pour effet de réduire la clairance des lipoprotéines riches en triglycérides. Quelques rares mutations affectant la structure et l'expression de l'ApoAI ont été identifiées qui peuvent être responsables d'une diminution du taux de cholestérol HDL et également d'une activité cofacteur de la LCAT anormale. La maladie de Tangier pour sa part, qui est caractérisée par un déficit très important des HDL, ne semble pas être associée à une anomalie de l'ApoAL. 
De nombreux polymorphismes au niveau du complexe ApoAI-CIIIAIV ont été décrits ; le Tableau III en donne une liste avec la fréquence de l'allèle rare pour chacun d'eux. Parmi ces polymorphismes, le polymorphisme SstI situé dans la partie 3 ' non codante du gène de l'ApoCIII [18-28] et le polymorphisme PstI situé en 3' de l'ApoAI [21, 28-30] ont été particulièrement étudiés (Tableaux IV et $V$ ). Les résultats montrent que $\mathrm{AI} / \mathrm{SstI}$ est associé à des anomalies du taux de triglycérides de manière assez cohérente, alors que l'association avec l'athérosclérose est moins convaincante. Il est intéressant de remarquer que quelques études ont montré une association entre l'allèle rare du polymorphisme PstI et des taux bas de cholestérol HDL et d'ApoAI [29]. Cet allèle pourrait donc être associé à un risque accru d'athérosclérose, ce qui semble confirmé par une autre étude (Wile et al., non publié, cité dans [17]).

L'apolipoprotéine AII. L'ApoAII est présente en quantité notable sur les HDL. Outre son rôle structural, elle semble activer la lipase hépatique et modifier l'activité de la LCAT. Un polymorphisme MspI, situé au niveau d'une séquence Alu répétée en 3' du gène de l'ApoAII, paraît associé au taux d'ApoAII circulant. L'allèle rare est présent chez $20 \%$ des sujets étudiés et les sujets homozygotes pour cet allèle ont un niveau d'ApoAII nettement augmenté par rapport aux autres sujets [31].

L'apolipoprotéine B. La structure du gène de l'ApoB est très différente de celle des autres apolipoprotéines; pourtant, il existe des similarités de séquences et, bien qu'il soit impossible actuellement de dire s'il s'agit de vraies homologies, il se pourrait que le gène de l'ApoB soit apparenté aux gènes des Apo AI, AIV et E [15].

\begin{tabular}{|c|c|c|c|}
\hline \multicolumn{4}{|c|}{$\begin{array}{l}\text { Tableau IV } \\
\text { PHISME ApoAI-CIII/SstI } \\
\text { OBSERVÉES AVEC LES LIPIDES } \\
\text { ET L'ATHÉROSCLÉROSE }\end{array}$} \\
\hline Référence & $\begin{array}{c}\text { Effectifs } \\
\text { des groupes* }\end{array}$ & $\begin{array}{c}\text { Association } \\
\text { avec les lipides }\end{array}$ & $\begin{array}{l}\text { Association avec } \\
\text { I'athérosclérose } \\
\text { et ses } \\
\text { complications }\end{array}$ \\
\hline Rees [18] & $35 / 73^{1}$ & oui & \\
\hline Rees [19] & $68 / 38 / 61^{2}$ & & oui \\
\hline Ferns [20] & $48 / 47^{3}$ & & oui \\
\hline Deeb [21] & $140 / 101^{3}$ & non & oui \\
\hline Rees [22] & $40 / 35^{1}$ & oui & non \\
\hline Hegele $^{a}$ & $66 / 66^{3}$ & & non \\
\hline Trembath [23] & $47 / 20 / 35^{4}$ & oui & \\
\hline Henderson [24] & $68 / 21 / 11 / 60^{5}$ & oui & \\
\hline Aalto-Setälä [25] & $29 / 20 / 39 / 61^{6}$ & oui & non \\
\hline Hayden [26] & $53 / 38^{7}$ & oui & \\
\hline Satoh [27] & $69 / 82^{3}$ & & non \\
\hline Paulweber [28] & $106 / 116^{3}$ & non & non \\
\hline
\end{tabular}

* Description des groupes: 1. patients hypertriglycéridémiques/témoins; 2. patients coronarographiés : pas de lésion/lésion ${ }^{+} /$lésion $^{++} ; 3$. patients coronariens/témoins; 4. patients diabétiques non insulinodépendants avec ou sans cardiopathies ischémiques et témoins non diabétiques; 5 . hypercholesterolémiques/hypertriglycéridémies/types III/témoins; 6 . hypertriglycéridémies familiales ou non familiales/patients coronariens/témoins; 7 . sujets hyperchlolestérolémiques/témoins. a. non publié, cité dans Humphries [17].
Le gène de l'ApoB est composé de 29 exons et de 28 introns qui codent pour une protéine de 4536 acides aminés. L'ApoB est le composant principal des LDL, des VLDL et des chylomicrons. Elle joue un rôle important dans la sécrétion de ces lipoprotéines et dans leur structure. De plus, ses capacités à se lier au récepteur des LDL lui confèrent un rôle essentiel dans la pénétration intracellulaire et le catabolisme du cholestérol. Deux espèces d'ApoB existent chez l'homme. L'une, l'ApoBl00, est une molécule de $550 \mathrm{kDa}$ (kilodalton) synthétisée par le foie qui participe à la formation des VLDL; l'autre, l'ApoB48; est une molécule de 210 kDa synthétisée dans l'intestin qui intervient dans la sécrétion des chylomicrons. Plusieurs approches ont permis de montrer que les deux protéines dérivent du même gène (par exemple, elles portent toutes deux sur leur partie homologue, les mêmes polymorphismes). La différence de longueur des deux molécules résulte d'une modification post- ou co-transcriptionnelle d'un seul nucléotide, avec pour conséquence l'apparition d'un codon stop en position 2153.

De nombreuses études ont tenté de relier la présence de polymorphismes de restriction du gène de l'ApoB à des anomalies lipidiques et à l'athérosclérose [21, 32-38]. Deux polymorphismes paraissent particulièrement intéressants. Un polymorphisme EcoRI qui affecte la séquence des acides aminés de la protéine mature au codon 4154 (Glu $\rightarrow$ Lys) et un polymorphisme $\mathrm{XbaI}$ dont nous avons déjà parlé. Les résultats du Tableau VI montrent des contradictions d'une étude à l'autre qui peuvent résulter soit d'une puissance insuffisante des études en terme d'effectifs, soit d'une hétérogénéité entre les populations étudiées. Néanmoins, il apparaît de manière assez cohérente que le polymorphisme XbaI est associé à des troubles lipidiques, tandis que le polymorphisme EcoRI semble associé à la survenue de troubles liés à l'athérosclérose.

Très récemment, l'utilisation d'un anticorps monoclonal (MB47), spécifique de la région de l'ApoB se liant au récepteur des $\mathrm{LDL}$, a permis de repérer des sujets hyporéactifs pour cet anticorps et ayant un taux de 


\begin{tabular}{|c|c|c|c|}
\hline \multicolumn{4}{|c|}{$\begin{array}{c}\text { POLYMORPHISMES ApoAI/PstI } \\
\text { ASSOCIATIONS OBSERVÉES AVEC LES LIPIDES } \\
\text { SANGUINS ET L'ATHÉROSCLÉROSE }\end{array}$} \\
\hline Référence & $\begin{array}{c}\text { Effectifs } \\
\text { des groupes }\end{array}$ & $\begin{array}{c}\text { Association avec } \\
\text { les lipides }\end{array}$ & $\begin{array}{c}\text { Association avec } \\
\text { l'athérosclérose } \\
\text { et ses } \\
\text { complications }\end{array}$ \\
\hline Ordovas [29] & $88 / 123$ & oui & oui \\
\hline Debs [21] & $140 / 114$ & non & non \\
\hline Hegele $^{c}$ & $76 / 76$ & & non \\
\hline Kessling [30] & $109^{b}$ & non & \\
\hline Paulweber [28] & $106 / 116$ & oui & non \\
\hline Wile $^{c}$ & $140 / 110$ & & oui \\
\hline
\end{tabular}

a. coronariens/témoins; $b$. sujets sélectionnés de manière à avoir un large éventail de valeurs de HDLC; $c$. non publié, cité dans Humphries [17].

\begin{tabular}{|c|c|c|c|}
\hline \multicolumn{4}{|c|}{$\begin{array}{c}\text { Tableau VI } \\
\text { POLYMORPHISMES ApoB/Xbal et ApoB/EcoRI } \\
\text { ASSOCIATIONS OBSERVÉES AVEC LES LIPIDES } \\
\text { SANGUINS ET L'ATHÉROSCLÉROSE }\end{array}$} \\
\hline Référence & $\begin{array}{c}\text { Effectifs } \\
\text { des groupes* }\end{array}$ & $\begin{array}{c}\text { Association avec } \\
\text { les lipides }\end{array}$ & $\begin{array}{l}\text { Association avec } \\
\text { I'athérosclérose } \\
\text { et ses } \\
\text { complications }\end{array}$ \\
\hline \multicolumn{4}{|c|}{ ApoB/Xbal } \\
\hline $\begin{array}{l}\text { Hegele [32] } \\
\text { Berg [33] } \\
\text { Deeb [21] } \\
\text { Law [34] } \\
\text { Talmud [35] } \\
\text { Ferns [36] } \\
\text { Aburatani [37] } \\
\text { Monsalve [38] } \\
\text { Gallacher }^{\mathrm{a}} \\
\text { Paulweber }^{\mathrm{a}}\end{array}$ & $\begin{array}{l}81 / 84^{1} \\
56^{2} \\
117 / 102^{1} \\
83^{2} \\
133 / 62^{3} \\
74 / 60^{1} \\
76 / 26 / 80^{4} \\
205 / 139^{5} \\
124 / 186^{1} \\
106 / 118^{1}\end{array}$ & $\begin{array}{l}\text { non } \\
\text { oui } \\
\text { oui } \\
\text { oui } \\
\text { oui } \\
\text { non } \\
\text { non }\end{array}$ & $\begin{array}{l}\text { non } \\
\text { non } \\
\text { oui } \\
\text { non } \\
\text { non }\end{array}$ \\
\hline \multicolumn{4}{|c|}{ ApoB/EcoRI } \\
\hline $\begin{array}{l}\text { Hegele [32] } \\
\text { Deeb [21] } \\
\text { Gallacher }^{\mathrm{a}} \\
\text { Paulweber }^{\mathrm{a}} \\
\text { Talmud [35] } \\
\text { Ferns [36] } \\
\text { Monsalve [38] }\end{array}$ & $\begin{array}{l}84 / 84^{1} \\
108 / 123^{1} \\
124 / 186^{1} \\
106 / 118^{\prime} \\
133 / 62^{3} \\
74 / 60^{1} \\
205 / 139^{5}\end{array}$ & non & $\begin{array}{l}\text { oui } \\
\text { non } \\
\text { oui } \\
\text { oui }\end{array}$ \\
\hline
\end{tabular}

* description des groupes: 1. coronariens/témoins; 2. sujets sains; 3. dyslipidémiques/témoins; 4. hyperlipidémiques/coronariens/témoins; 5. artériopathie des membres supérieurs/témoins. a. non publié, cité dans Humphries [17].

$\mathrm{m} / \mathrm{s} n^{\circ} 6$ vol. 5 , juin 89

cholestérol LDL augmenté. Par séquençage, il a été possible de montrer que l'anomalie en cause était une mutation Arg $\rightarrow$ Lys en position 3500 de l'ApoB mature. Dans la mesure ou la séquence du gène de l'ApoB était connue, il a été possible d'amplifier la région incriminée par la technique PCR [Innerarity et al., non publié], puis - par hybridation avec des oligonucléotides spécifiques de l'allèle muté et de l'allèle sauvage - de rechercher la présence de la mutation chez un grand nombre de sujets. Sept homozygotes pour la mutation ont été identifiés dans une population nord-américaine de 845 sujets. S'il est vérifié que ces individus n'étaient pas apparentés, cette population pourrait comprendre environ $15 \%$ d'hétérozygotes. Ce pourcentage important fait que, même si l'anomalie lipidique présente chez les nétérozygotes est modeste, son impact sur la population peut être important.

\section{Commentaires et conclusions}

Comme nous l'avons signalé dans l'introduction, la définition du polymorphisme repose en partie sur la fréquence relativement grande de ses allèles dans la population. C'est la raison pour laquelle nous avons peu parlé des variants rares et des études familiales sur lesquelles repose leur caractérisation.

Les polymorphismes des apolipoprotéines ont fait l'objet d'un nombre important de travaux au cours de ces dernières années et l'étude des polymorphismes des gènes codant pour ces apolipoprotéines, en particulier par la technique de Southern, a pris le pas sur les autres approches. Les résultats de tous ces travaux sont difficiles à résumer ; certaines associations paraissent établies de manière convaincante, alors que d'autres font apparaître des différences considérables d'une étude à l'autre. Bien sûr, pour expliquer ces différences, il est possible d'arguer de la grande hétérogénéité des fréquences alléliques d'une population à une autre [39] qui peut non seulement expliquer des différences de résultats entre études, mais aussi des associations à l'intérieur d'une même étude lorsque les malades et les 


\begin{tabular}{|c|c|c|c|}
\hline \multicolumn{4}{|c|}{ Classification des dyslipoprotéinémies } \\
\hline Type & Cholestérolémie & Triglycéridémie & Lipoprotéines \\
\hline $\mathbf{I}$ & $\begin{array}{l}\text { normale ou } \\
\text { modérément élevée }\end{array}$ & très élevée & chylomicrons +++ \\
\hline IIa & élevée & normale & $\mathrm{LDL}+++$ \\
\hline IIb & élevée & $\begin{array}{l}\text { modérément } \\
\text { élevée }\end{array}$ & $\begin{array}{l}\mathrm{LDL}+++ \\
\mathrm{VLDL}+\end{array}$ \\
\hline III & élevée & $\begin{array}{l}\text { modérément ou } \\
\text { très élevée }\end{array}$ & $\begin{array}{l}\text { VLDL, LDL de } \\
\text { composition } \\
\text { anormale }\end{array}$ \\
\hline IV & $\begin{array}{l}\text { modérément } \\
\text { élevée }\end{array}$ & $\begin{array}{l}\text { modérément ou } \\
\text { très élevée }\end{array}$ & $\mathrm{VLDL}+++$ \\
\hline $\mathbf{V}$ & $\begin{array}{l}\text { modérément } \\
\text { élevée }\end{array}$ & très élevée & $\begin{array}{l}\text { chylomicrons }++ \\
\text { VLDL }+++\end{array}$ \\
\hline
\end{tabular}

témoins ne sont pas représentatifs de la même population. Nombre de ces différences peuvent également être expliquées par l'hétérogénéité des maladies étudiées et par la puissance insuffisante des études en terme d'effectifs.

L'intérêt porté aux polymorphismes dans l'étude des maladies fréquentes, telles les dyslipoprotéinémies ou l'athérosclérose, repose implicitement sur l'hypothèse que des polymorphismes qui, isolés ou dans des conditions favorables, n'ont pas d'effet délétère, peuvent, en conjonction ou en présence d'un environnement défavorable, déterminer la résistance ou la susceptibilité à ces maladies. Les études visant à évaluer cette hypothèse doivent donc porter sur des effectifs importants de malades et de témoins et doivent explorer chez les mêmes sujets une série de polymorphismes affectant la même férentes. Aucune étude jusqu'à présent n'a rempli ces conditions.

En outre, de nouvelles techniques de lisées. La recherche sur les polymorphismes va bénéficier dans les mois et annéesà venir des techniques d'amplification génique et de séquençage, nous y avons fait allusion dans la discussion sur la mutation du site 3500 de l'ApoB. Le recoursà ces techniques devrait se généraliser et permettre d'éviter le gros inconvénient des polymorphismes de restriction - qui ne sont que des marqueurs et comme tels sont affectés d'une spécificité et d'une sensibilité parfois faibles et en règle générale difficiles à évaluer. Avec ces techniques, l'étude de régions candidates de dimension limitée à quelques centaines de bases et jouant un rôle complémentaire dans le métabolisme des lipides pourrait permettre une approche directe des interactions géniques biologie moléculaire doivent être utirégion et des régions candidates dif-

\section{Summary}

Genetic polymorphisms of apolipoproteins

In the recent years, the polymorphisms of apolipoproteins (Apo) have been much investigated; in particular with the help of many restriction polymorphisms (RFLP) which were found on or near the genes coding for these proteins. Associations between polymorphisms of ApoAI, AII, AIV, B, CIII and E and lipids abnormalities or coronary heart disease have been demonstrated in case-control studies. The reported results suggest that a small number of polymorphisms with frequent alleles might have a much greater impact on lipid abnormalities than rare variants determining serious familial diseases such as familial hypercholesterolemia. The joint effect of these polymorphisms could explain most of the genetic dyslipidaemia. To test this hypothesis, large studies are needed and new approaches such as PCR (polymerase chain reaction) amplifications and sequencing of strategic regions of candidate genes will be used.

\section{Remerciements}

Je remercie Pascale Benlian (en particulier pour son aide dans la conception du Tableau III), Claudine Junien, Gérard Cuny, Philippe Douste-Blazy, Pierre Ducimetière, Gérard Roizes et Florent Soubrier pour leur collaboration et pour d'utiles discussions. 